\title{
Wave Function Scarring Effects in Open Ballistic Quantum Cavities
}

\author{
R. AKIS and D. K. FERRY* \\ Nanostructures Research Group, Center for Solid State Electronics, Arizona State University, Tempe, AZ 85287
}

\begin{abstract}
Numerical simulations have been carried out of the magneto-transport and corresponding wave functions of two quantum dot structures, namely the stadium and the Sinai billiard. In our simulations, the Schrödinger equation is mapped onto a tight-binding lattice by replacing derivatives by finite differences, and the conductance is calculated via the Landauer formula following the application of an iterative technique to translate across the structure. In both structures, many of the resonance features in the transport show scarring, that is, the amplitude of the corresponding wave functions is highly concentrated along underlying periodic classical orbits. Our analysis indicates that certain periodicities evident in the magneto-transport can be associated with particular orbits.
\end{abstract}

Keywords: Transport, numerical simulation, quantum simulation, chaos, periodic orbits, finite differences

\section{INTRODUCTION}

Semiconductor billiards have recently attracted considerable interest as a novel probe of transport and quantum chaos. The devices examined typically consist of a central mesoscopic cavity, connected to external reservoirs by a pair of quantum point contacts through which current is measured. The lithographic size of these dots is usually made much smaller than the mean free path, so that transport is ballistic. While it has been suggested that multiple billiard scattering from cavity walls will induce chaotic behavior [1], recent studies of rectangular billiards have established the basic regular nature of the orbits [2]. In stadium billiards, it is still thought that chaotic behavior is induced, even in open structures $[3,4]$. However, recent experiments on stadium shaped dots suggest that their electrical properties are dominated by a characteristic periodicity in their magnetoconductance $[3,5]$. In the earlier study, it was tentatively suggested that this periodicity arises from the remnants of regular, semi-classical orbits within the dots [3]. In order to investigate

*Corresponding author. Tel.: 602-965-2570, Fax: 602-965-8058, e.mail: ferry@asu.edu. 
this effect, we have modeled the dots by solving the quantum mechanical problem on a discrete lattice using a numerically stabilized variant of the transfer matrix approach [6]. The details of this method are summarized in the next section. The section following presents our theoretical results for the stadium as well as a comparison with experiment. Simulating dots of comparable size, we reproduce the periodicity of the fluctuations found experimentally. Moreover, we find that the wave functions of these structures can be heavily scarred by periodic orbits, with certain scars recurring periodically in magnetic field, in good correspondence with selected peaks in the power spectra. In the next section, we consider the Sinai billiard, which consists of a nominal square ballistic quantum dot with a circular anti-dot in the center and is another structure whose classical analog is chaotic. Recent experiments [7] have shown self-similar (fractal) behavior in the magnetoconductance of such structures, appearing in a series of peaks in the resistance. Not only do our simulations show indications of self-similarity on scales similar to that observed experimentally, but that this behavior can in fact coexist with the periodic scarring effect associated with regular orbits. The paper concludes with a summary.

\section{METHOD OF CALCULATION}

As displayed in Figure 1, the typical situation is one in which ideal quantum wires, which extend outward to $\pm \infty$ are connected to the quantum dot, a stadium in this case. This problem can be solved by using an iterative method [6] applied to the discretized version of the Schrödinger equation, obtained by keeping terms up to first order in the approximation of the derivative:

$$
\left(E_{F}-\mathbf{H}_{j}\right) \psi_{j}+\mathbf{H}_{j, j-1} \psi_{j-1}+\mathbf{H}_{j, j+1} \psi_{j+1}=0
$$

where $\psi_{j}$ is a $M$-dimensional vector containing the amplitudes of the $j$ th slice. The problem is solved on a square lattice of lattice constant $a$ with the

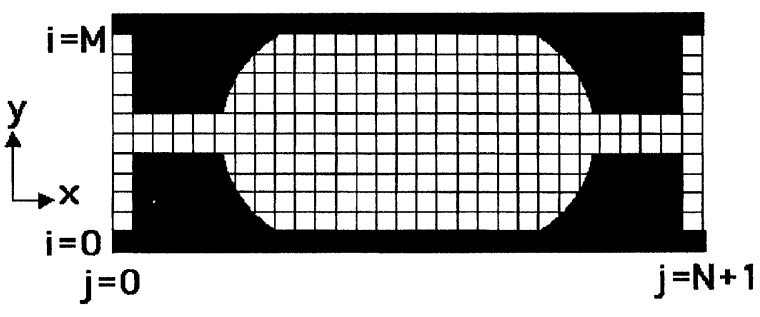

FIGURE 1 The geometry of one of the quantum dots considered in this study. The grid represents the underlying mesh on which the calculations are performed, though in practice the grid is much finer that that shown here.

wires extending $M$ lattice sites across in the $x$ direction and the region of interest being broken down into a series of slices along the $y$ direction. In this equation, the $\mathbf{H}_{j}$ matrices represent Hamiltonians for individual slices and the matrices $\mathbf{H}_{j, j-1}$ and $\mathbf{H}_{j, j+1}$ give the inter-slice coupling. By approximating the derivative, the kinetic energy terms of Schrödinger's equation get mapped onto a tight-binding model with $t=-\hbar^{2} / 2 m^{*} a^{2}$ representing nearest neighbor hopping. The potential simply adds to the on site energies. Assuming a flux incident from the left, this equation can be used to derive a transfer matrix which allows us to translate across the system and thus calculate the transmission coefficients which enter the Landauer-Büttiker formula to give the conductance. Transfer matrices however are made unstable due to exponentially growing and decaying contributions of the evanescent modes. This difficulty can be overcome by performing some clever matrix manipulations and calculating the transmission by a iterative procedure rather than just multiplying transfer matrices together [6]. The amplitudes of the wave functions at specific values of $x$ and $y$ can be found easily by backward substitution after the iteration is performed.

\section{THE STADIUM}

The correspondence between periodicities in the conductance and scarring effects was first suggested as a possibility in the context of stadium 
shaped quantum dots by Marcus et al. [3]. Provided an ensemble average was not performed, they found that a Fourier analysis of their conductance fluctuations revealed the presence of strong peaks at a few discrete frequencies. The stadium is of particular interest, since its closed, classical analog is well known to be chaotic (it should be noted that a closed quantum stadium structure has also been achieved [7]). As such, one might expect the behavior to reflect ergodicity, or phase space filling. Such behavior is only recovered after ensemble averaging over many conductance traces, each obtained by altering the gate voltage $[3,4]$. Importantly, such averaging has the effect of returning the dot, and its point contact leads, to the classical regime, since the quantization is averaged out, and the proper quantum behavior is masked.

The results of a Fourier analysis of a single conductance trace are shown in Figure 2(a) as curve (a), taken from Ref. [3]. In the inset is a micrograph of the actual dot on which the experiments were performed. As shown, the input and output leads were at right angles to each other. The dot size was approximately $0.4 \times 1.0 \mu \mathrm{m}$ and the electron density was $3.6 \times 10^{11} \mathrm{~cm}^{-2}$. Curve (b) is the Fourier power spectrum obtained from our simulation of a similar sized dot (after accounting for depletion). Both the experiment and the simulation show strikingly similar harmonic content, with well defined peaks at $\mathrm{f} \sim 20,33$ and $65 \mathrm{~T}^{-1}$ in both cases. For the simulation, four modes were allowed to propagate in the leads. In addition to the periodicity, we see scarring effects. Figure 2(b) shows a representative example, a wave function scarred by an underlying "bow-tie" shaped classical orbit, which occurs at $B=0.229 \mathrm{~T}$. Unfortunately, while other scars do appear, it is difficult to establish a connection between any scar and a particular power spectrum peak, as similar looking scars can be resolved only at a very few other values of field.

Figure 3 shows an example in which the stadium has centrally aligned leads, dimensions of $0.4 \mu \mathrm{m} \times$ $0.8 \mu \mathrm{m}$ and an electron density of $4 \times 10^{11} \mathrm{~cm}^{-2}$. (a)

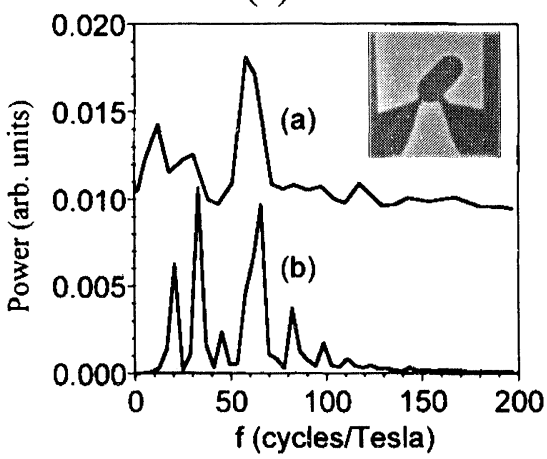

(b)

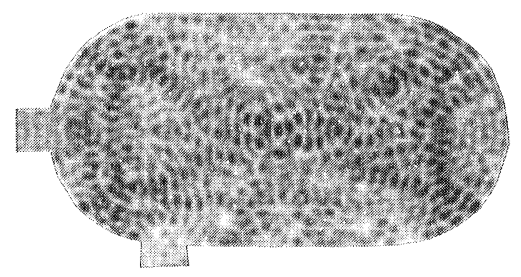

FIGURE 2 In panel (a), the Fourier power spectrum of the conductance fluctuations is plotted. The curve labeled (a) is for the experimental stadium dot shown in the inset (from Ref. [3], with permission). Curve (b) is the result of the quantum simulation. Panel (b) show a "bow-tie" scar that appears in the stadium wave function. Probability density $\left(|\psi(x, y)|^{2}\right)$ versus $x$ and $y$ plotted here, with higher amplitude corresponding to darker shading.

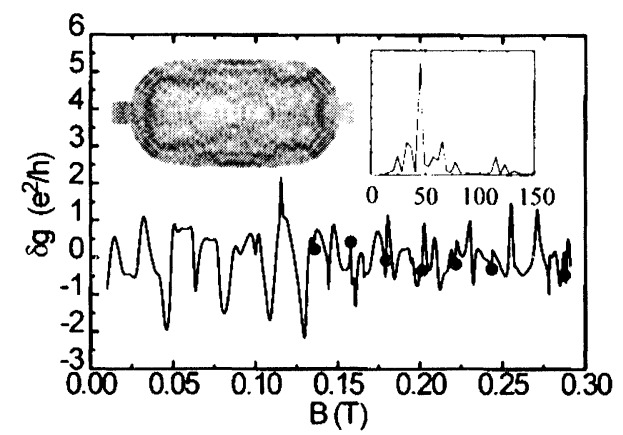

FIGURE 3 Conductance fluctuations, $\delta \mathrm{g}$, are plotted as a function of magnetic field for a stadium quantum dot with aligned leads. The right inset shows the corresponding power spectrum. The left inset shows a "whispering gallery" scar that recurs at the points indicated by the solid circles.

Here we plot the conductance fluctuations for this configuration and the corresponding power spectrum is shown in the left inset. A single peak at 47 
$\mathrm{T}^{-1}$ clearly dominates. The corresponding magnetic field period is $0.021 \mathrm{~T}$, in good agreement with the spacings between successive minima in the fluctuations. The solid circles correspond to values of $B$ where a "whispering-gallery" scar was observed in the wave function. An example of such a scar is shown in the left inset, which corresponds to $B=0.288 \mathrm{~T}$. The quantization of modes in the contacts causes the electrons to be injected in collimated beams directed at well defined angles [2], and it is this effect that is responsible for exciting the orbit seen here. The "whisperinggallery" scars appear at very nearly periodic intervals, with the period also being $0.021 \mathrm{~T}$, thus a strong correspondence can be made between a particular power spectrum peak and a periodic orbit in this case.

\section{THE SINAI BILLIARD}

As mentioned in the introduction, the Sinai billiard is another example in which the classical dynamics is expected to be chaotic, at least in the closed structure. The experimental observation of "self-similarity" in the magnetoresistance of such structures has been recently reported [8]. Selfsimilar or fractal behavior has long been associated with chaos. We have performed simulations of Sinai billiard structures, with configurations similar to the experimental ones, with a quantum dot $1.0 \mu \mathrm{m}$ square, an anti-dot in the center, two leads in the bottom left-hand size corner (refer to Fig. 4(c)) and an electron density of $2.3 \times 10^{11} \mathrm{~cm}^{-2}$. Figure 4(a) shows a resistance trace obtained from such a simulation, with the leads adjusted to support four propagating modes, and the central anti-dot approximately $0.2 \mu \mathrm{m}$ in radius. We show this example because, first of all, it shows indications of the self-similar behavior seen experimentally. A blow-up of the central region of Figure 4(a), is displayed in Figure 4(b). Note that the basic form of two broad humps occurs on both scales. The peak of the hump in (a) occurs at $\sim 10 \mathrm{mT}$ and in (b) at $\sim 0.5 \mathrm{mT}$, indicating a
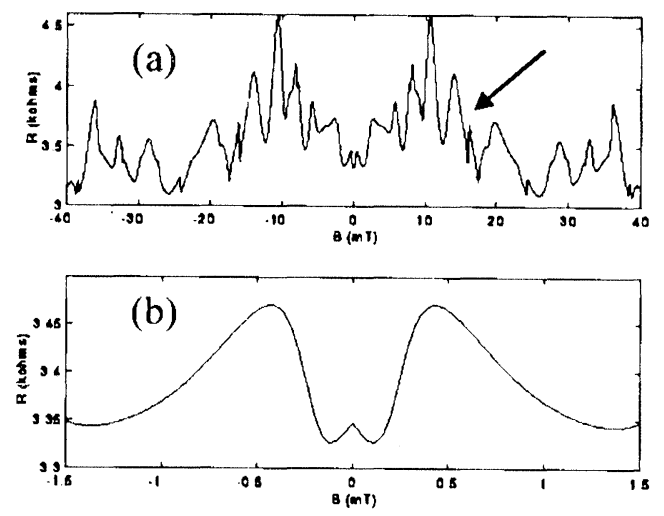

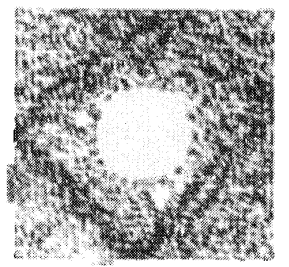

(c)

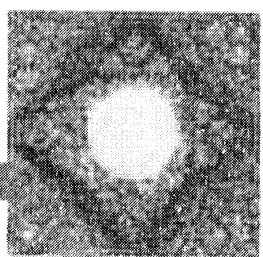

(d)
FIGURE 4 (a) A resistance trace obtained from a simulation of a Sinai billiard, with input and output leads are in the bottom left hand corner of the structure (these are seen clearly in (d)). (b) A blow-up of the central region of shown in (a). (c) The scarred wave function at $B=16 \mathrm{mT}$, which corresponds to the resonance indicated by the arrow in the top frame. (d) Another diamond scar from the periodic series, this one at $B=49 \mathrm{mT}$.

scaling factor of $\sim 20$, which is in agreement with that observed experimentally [8]. Secondly, despite this apparent self-similar behavior, this example also shows periodic scarring effects similar to that observed for stadium. Figure 4(c) shows the scarred wave function $B=16 \mathrm{mT}$, which corresponds to the resonance indicated by the arrow in Figure 4(a). Note the distorted diamond-shaped periodic orbit reflected by the wave function. Similar scarred wave functions appear at intervals of $\sim 8 \mathrm{mT}$. Figure $4(\mathrm{~d})$ shows another diamond scar from this periodic series, this one at $B=49 \mathrm{mT}$. As in the stadium, the excitation of this particularly periodic orbit can be attributed in large part due to the collimation effect on the input leads. In fact, similar scars have been observed in simple square quantum dots [2]. What is interesting however in the Sinai billiard is that, the 
circular anti-dot in the center can in fact cause a "shadow" effect, which can make configuration appear more regular than the simple regular dot. This is most apparent when the collimated beam exiting the left contact just grazes the anti-dot and is indicated by the periodic appearance of many well defined scars. For example, in a $0.3 \mu \mathrm{m}$ square dot, four examples of a diamond shaped orbit were observed at periodic intervals, with the periodic scarring effects becoming much weaker as the dot is made larger [2]. In contrast, with a much larger Sinai billiard dot, we have seen the diamond pattern recur as many as six times. We shall give a much fuller account of these results elsewhere.

\section{SUMMARY}

Despite the fact that the closed, classical analogs of the structures we have studied are know to be chaotic, we have found that regular periodic orbits strongly influence the magneto-transport of both the stadium and Sinai billiard structures. Evidence for these orbits is seen in scarred wave functions, which can recur periodically in magnetic field. In both cases, the quantization of modes in the leads is crucial in exciting the particular orbits evident in the scarred wave functions, as this quantization causes the electrons to be injected in collimated beams directed at well defined angles. For the stadium in particular, the scarring periodicities show good correspondence with power spectrum peaks, providing strong evidence that the periodicities evident in the magneto-conductance fluctuations are in fact tied to specific periodic orbits. Moreover, we obtain power spectra that agree well with the experimental observations. In the Sinai billiard, we see indications of the self-similar behavior observed experimentally coexisting with the periodic scarring behavior. The actual origin of the self-similar behavior in this structure requires further study. However, in this regard, it should be mentioned that the results of a semi-classical analysis have lead to the prediction of fractal conductance fluctuations in chaotic cavities, no- tably for cases in which there is a mixed (chaotic and regular) phase space [9].

\section{Acknowledgements}

The authors would like to express their appreciation to C.M. Marcus for allowing us to use his data in this paper and R. P. Taylor for useful discussions. The work was supported by ONR and DARPA.

\section{References}

[1] Jalabert, R. A., Baranger, H. U. and Stone, A. D. (1990). "Conductance Fluctuations in the Ballistic Regime: a Probe of Quantum Chaos?", Phys. Rev. Lett., 65, 2442.

[2] Akis, R., Ferry, D. K. and Bird, J. P. (1996). "Magnetotransport Fluctuations in Regular Semiconductor Ballistic Quantum Dots", Phys. Rev. B., 54, 17705.

[3] Marcus, C. M., Rimberg, A. J., Westervelt, R. M., Hopkins, P. F. and Gossard, A. C. (1992). "Conductance Fluctuations and Chaotic Scattering in Ballistic Microstructures", Phys. Rev. Lett., 69, 506.

[4] Chang, A. M., Baranger, H. U., Pfeiffer, L. N. and West, K. W. (1994). "Weak Localization in Chaotic versus Nonchaotic Cavities: a Striking Difference in Lineshape", Phys. Rev. Lett., 73, 2111.

[5] Okubo, Y., Ochiai, Y., Bird, J. P., Ferry, D. K., Ishibashi, K., Aoyagi, Y. and Sugano, T. (1997). "Magnetically Induced Supression of Phase Breaking in Ballistic Mesoscopic Billiards", Phys. Rev. B., 55, 1368.

[6] Usuki, T., Saito, M., Takatsu, M., Kiehl, R. A. and Yokoyama, N. (1995). "Numerical Analysis of Ballistic Electron Transport in Magnetic Fields by Using a Quantum Point Contact and a Quantum Wire", Phys. Rev. B., 52, 8244.

[7] Harbury, Y. and Porod, W. (1996). "Elastic Scattering Theory for Electronic Waves in Quantum Corrals", Phys. Rev. B., 53, 15455.

[8] Taylor, R. P. et al. (1997). "Self-Similar Magnetoresistance of a Semiconductor Sinai Billiard", Phys. Rev. Lett., 78, 1952.

[9] Ketzmerick, R. "Fractal Conductance Fluctuations in Generic Chaotic Cavities", Phys. Rev. B., in press.

\section{Authors' Biographies}

Richard Akis received his bachelor's degree in physics from Lakehead University, Thunder Bay, Canada in 1986, where he won the Science Gold Medal. He received a Ph.D. in Physics in 1991 from McMaster University, Hamilton, Canada, in the area of superconductivity. For his postdoctoral 
work, he switched fields, and has been studying semiconductor nanostructures, initially at Simon Fraser University, Burnaby, Canada, where he was a NSERC postdoctoral fellow and subsequently at Concordia University, Montreal, Canada, where he was a research associate. Since 1995, he has been a faculty research associate at Arizona State University.

David K. Ferry is Regents' Professor of Engineering at Arizona State University. Prior to joining ASU in 1983, he was a faculty member at Colorado State University (1977-83) and Texas Tech University (1967--73), and worked at the Office of Naval Research (1973-77). He received his bachelor's and master's degrees in electrical engineering from Texas Technological College in 1962 and 1963, respectively, and the Ph.D. in electrical engineering from the University of Texas in 1966. He was a NSF postdoctoral fellow at the University of Vienna (1966-67). 

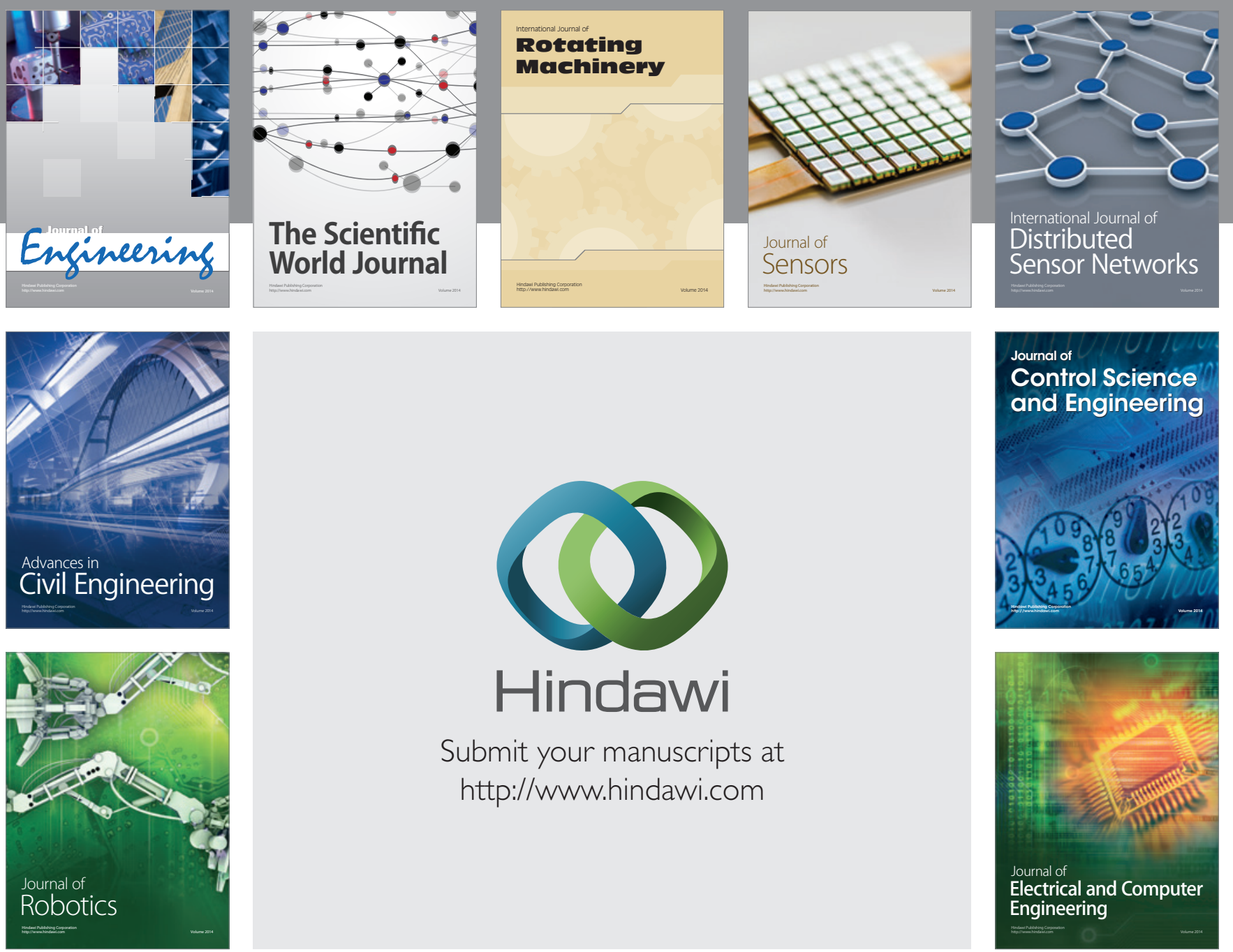

Submit your manuscripts at

http://www.hindawi.com
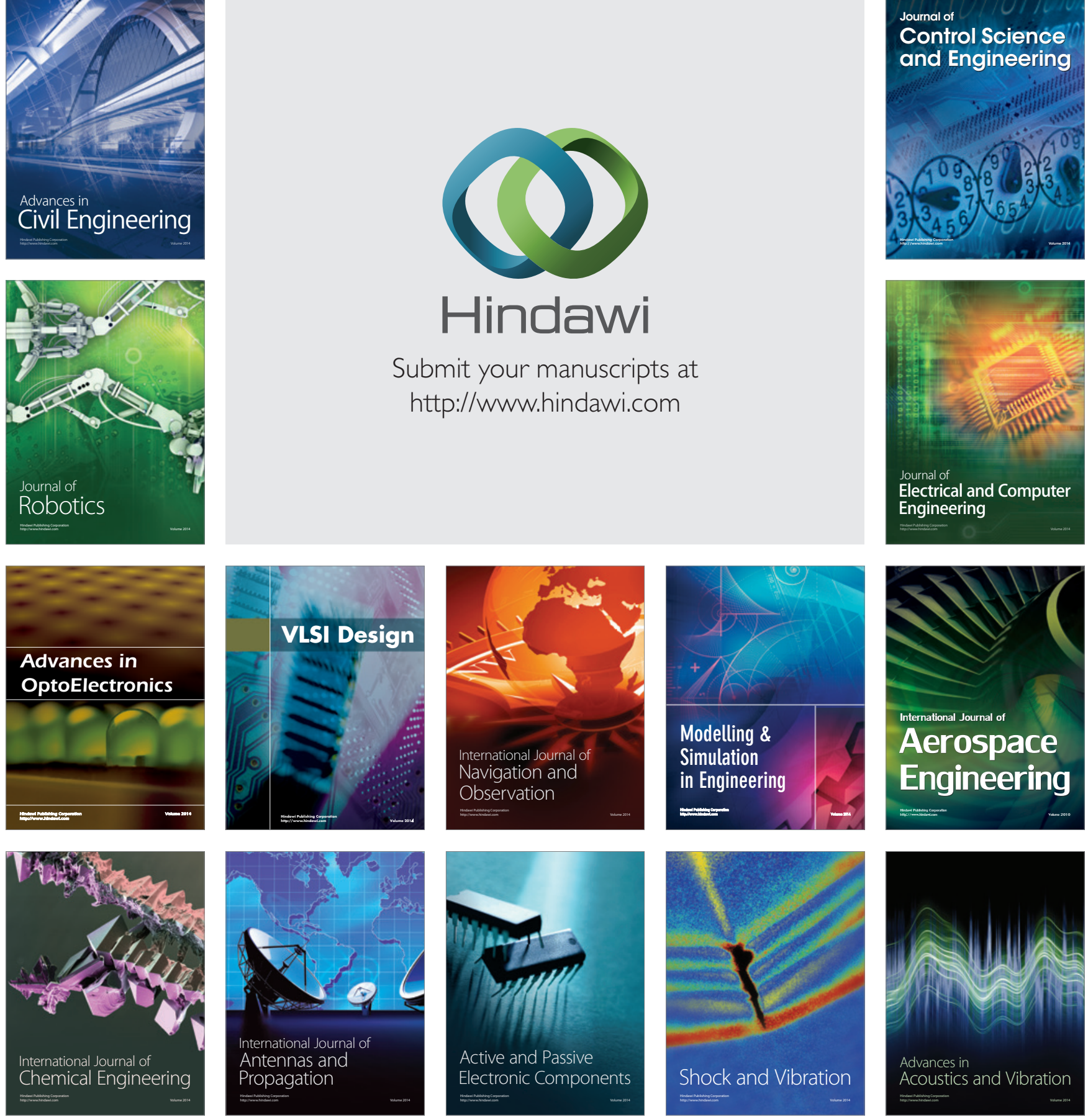